\title{
Politique
}

\section{La nouvelle analyse institutionnelle}

\section{Réjean Landry}

Numéro 6, automne 1984

La réforme des institutions politiques

URI : https://id.erudit.org/iderudit/040459ar

DOI : https://doi.org/10.7202/040459ar

Aller au sommaire du numéro

Éditeur(s)

Société québécoise de science politique

ISSN

0711-608X (imprimé)

1918-6584 (numérique)

Découvrir la revue

Citer cet article

Landry, R. (1984). La nouvelle analyse institutionnelle. Politique, (6), 5-32.

https://doi.org/10.7202/040459ar

Ce document est protégé par la loi sur le droit d'auteur. L'utilisation des services d'Érudit (y compris la reproduction) est assujettie à sa politique d'utilisation que vous pouvez consulter en ligne.

https://apropos.erudit.org/fr/usagers/politique-dutilisation/
Cet article est diffusé et préservé par Érudit.

Érudit est un consortium interuniversitaire sans but lucratif composé de l'Université de Montréal, l'Université Laval et l'Université du Québec à Montréal. Il a pour mission la promotion et la valorisation de la recherche. https://www.erudit.org/fr/ 


\title{
La nouvelle analyse institutionnelle*
}

\author{
Réjean Landry \\ Université Laval
}

La science politique est-elle en train de redevenir la science de l'étude des institutions? Alors que certains parlent du retour de l'institutionnalisme ${ }^{1}$, un des politologues les plus prestigieux, William Riker soutient que «we cannot study simply tastes and values, but must study institutions as well ${ }^{2}$. L'analyse de l'État de Richard Auster et Morris Silver ${ }^{3}$ et l'examen des rigidités sociales traitées dans le dernier ouvrage de Mancur Olson ${ }^{4}$ concernent essentiellement l'impact redistributif que les institutions exercent

* L'auteur tient à remercier les deux évaluateurs anonymes de la revue pour la qualité et l'utilité de leurs commentaires.

1. James F. Blumstein, "The Resurgence of Institutionalism », Journal of Policy Analysis and Management, 1 (1981), 129-132.

2. William H. Riker, "Implications from the Disequilibrium of Majority Rule for the Study of Institutions", dans Peter C. Ordeshook et Kenneth A. Shepsle (eds.), Political Equilibrium, (Boston, Mass.: Kluwer-Nijoff Publishing Co., 1982), 3-24. D'abord publié dans American Political Science Review, 74 (1980), 432-447. Kenneth A. Shepsle tente lui aussi de démontrer l'impact des institutions sur l'équilibre dans «The role of Institutional Structure in the Creation of Policy Equilibrium", dans Douglass W. Ray et Theodore J. Eismeir (eds.), Public Policy and Public Choice (Beverly Hills, Ca.: Sage Publications, 1979), 249-281.

3. Richard D. Auster et Morris Silver, The State as a Firm, Economic Forces in Political Development (Boston, Mass. : Martinus Nijhoff Publishing, 1979).

4. Mancur Olson, The Rise and Decline of Nations (New Haven, Conn.: Yale University Press, 1982). 
sur les individus et les groupes. D'après Charles Plott, l'analyse des choix sociaux soulève des difficultés découlant en partie de la nature illusoire des institutions. Son bilan de la théorie axiomatique des choix sociaux l'amène à conclure qu'il est nécessaire de formuler une «théorie des institutions révélées»:

«Because institutions specify the information and opportunities of process participants, predictions deduced from existing behavioral generalizations are very sensitive to assumptions about institutions. Without rather extensive knowledge about institutions, we cannot be confident of our analysis of the impact of those policies which would alter part of the institutional structure. Furthermore, at the basic scientific level we cannot distinguish between behavioral generalizations which are wrong because of a "misspecified value", the institutions, and those which are simply wrong'."

Cet appel à l'étude des institutions ne va pas sans difficulté. Il s'inscrit dans un contexte épistémologique qui considère le paradigme institutionnaliste comme démodé et dans un contexte historique marqué par une grande désillusion face aux institutions publiques. De plus, le retour à l'institutionnalisme soulève des difficultés méthodologiques et des choix de stratégies de recherche qui ne sont pas faciles à faire.

\section{Le contexte épistémologique}

La science politique repose sur deux traditions importantes, la plus ancienne postulant que les choix individuels dépendent des caractéristiques des institutions et, la plus récente, postulant que les goûts et les valeurs individuelles déterminent les choix des individus ${ }^{6}$. Le paradigme institutionnaliste, qui constitue le

5. Charles R. Plott, "Axiomatic Social Choice Theory: An Overview and Interpretation", American Journal of Political Sicence, 20 (1976), 583.

6. Le mot paradigme est pris dans le sens large que lui attribue T.S. Kuhn, La structure des révolutions scientifiques, (Paris: Flammarion, 1972), ouvrage publié initialement en anglais en 1962. 
paradigme traditionnel de la science politique, a dominé l'avantscène jusque vers les années dix-neuf cent quarante, soit au moment de l'avènement de la révolution behavioriste.

Le paradigme institutionnaliste remonte au moins jusquà Aristote qui a accordé une grande importance à la description des constitutions de son époque. À partir du XIX $\mathrm{XI}^{\mathrm{e}}$ siècle, on commence à opposer les termes institutions et constitutions en associant le terme institution à l'idée de structure organisationnelle de l'action collective. C'est ainsi que les libéraux réclament des constitutions qui modifieraient les institutions alors que les conservateurs s'y opposent en clamant la supériorité des institutions "naturelles» qui ne faisaient que protéger leurs intérêts. D'après Maurice Duverger, le programme de recherche du paradigme institutionnaliste déborde aujourd'hui l'étude des institutions réglementées par le droit pour s'étendre aux partis politiques, aux groupes d'intérêt, ainsi de suite. De plus, ajoute-t-il,

"même les institutions politiques réglementées par le droit, celles qu'établissent la Constitution ou les lois qui la complètent, ne doivent plus être étudiées uniquement sous l'angle juridique; désormais on doit essayer de définir dans quelle mesure elles fonctionnent conformément au droit et dans quelles mesures elles y échappent ${ }^{7}$."

Le postulat central du paradigme institutionnaliste est que les institutions déterminent les décisions des individus et les caractéristiques des biens issus de l'action collective. D'après William Riker, la version la plus poussée de l'institutionnalisme classique renvoie à la théorie marxiste lorsqu'elle soutient que les institutions économiques déterminent non seulement les comportements des individus mais aussi la direction de l'histoire de l'humanité ${ }^{8}$.

7. Maurice Duverger, Institutions politiques et droit constitutionnel, (Paris: Presses universitaires de France, Collection Thémis, 1968), 7-8.

8. William H. Riker, "Implications From the Disequilibrium of Majority Rules for the Study of Institutions", 5. 
Depuis une trentaine d'années, l'analyse des institutions a cédé le pas à l'étude de la culture politique, des comportements politiques et de l'opinion publique, focalisant ainsi de façon prédominante sur les goûts et les valeurs des individus, d'où l'appellation de paradigme behavioriste. Celui-ci diffère de l'institutionnalisme sous plusieurs aspects cruciaux: pour le behavioriste, l'institution constitue le contexte dans lequel se déroule l'interaction entre les individus. L'unité d'analyse du behavorisme renvoie donc à l'individu plutôt qu'à l'arrangement institutionnel. D'après les behavioristes les choix des individus s'expliquent non par les caractéristiques des institutions mais par les goûts et les valeurs des individus. Par ailleurs, le behaviorisme constitue une tentative pour expliquer de façon scientifique le choix des individus. Les empiristes ont eu recours à la quantification et à l'utilisation de sondages pour produire des généralisations empiriques. D'autre part, les tenants de la modélisation mathématique ont dérivé des théorèmes décrivant les choix collectifs découlant de diverses distributions des choix fondés sur les goûts et les valeurs des individus?. Les travaux empiriques et théoriques des behavioristes reposent sur le postulat implicite que les caractéristiques des institutions n'exercent aucun impact sur les choix des individus.

Le passage du paradigme institutionnaliste au paradigme behavioriste s'est opéré suivant le modèle de révolution scientifique décrit par Thomas Kuhn. Les groupes de politologues qui ont contribué à l'émergence du paradigme behavioriste ont réussi à renverser la domination des tenants du paradigme institutionnaliste sans considérer, dans le passage d'un paradigme à l'autre, l'effet cumulatif des connaissances.

9. À titre d'exemple, consulter Duncan Black, The Theory of Committees and Elections, (Cambridge: Cambridge University Press, 1957). La première partie du bilan de Charles Plott, "Axiomatic Social Choice Theory", constitue une autre illustration de cette stratégie de théorisation. 
Par contre, le passage que les politologues sont en train d'effectuer du paradigme behavioriste vers un paradigme qui réincorpore les arrangements institutionnels s'opère dans le cadre du développement d'un processus cumulatif des connaissances. Les connaissances deviennent cumulatives lorsque les découvertes produites à un moment donné dépendent de découvertes effectuées à un moment antérieur. Le développement cumulatif des connaissances n'est possible qu'en autant que les politologues adhèrent à des règles explicites concernant la formation et la transformation des connaissances. Lee Freese prétend que «toutes les sciences cumulatives utilisent des langages formels rigoureux auxquels adhèrent les scientifiques ${ }^{10}$. Selon lui, «la croissance systématique de la connaissance sociologique est ralentie non seulement à cause du manque de connaissances et de l'absence de consensus quant à la désirabilité du recours à des méthodes de formalisation mais aussi par le désaccord qui règne au sujet des propriétés que ces méthodes devraient posséder ${ }^{11}$.

De plus, un ensemble de connaissances est cumulatif seulement s'il exerce un effet cumulatif. Or un tel effet ne peut exister que si un ensemble donné de connaissances s'insère dans d'autres ensembles pour accroître le nombre de découvertes, ou, plus généralèment, le nombre d'explications et de prédictions fiables et valides. Le recours à des méthodes de formalisation mathématique a permis aux théoriciens de l'analyse des choix des individus de situer leurs résultats par rapport à des prédécesseurs dont il faut améliorer les explications et les prédictions. La formalisation mathématique des choix individuels fondée sur l'analyse des goûts et des valeurs des individus démontre essentiellement que les situations d'équilibre sont difficiles à atteindre et que les choix

10. Lee Freese, "The Problem of Cumulative Knowledge", dans Lee Freese (ed.), Theoretical Methods in Sociology: Seven Essays, (Pittsburgh, Penn.: The University of Pittsburgh Press, 1980), 21.

11. Lee Freese, "The Problem of Cumulative Knowledge», 22. 
des individus sont fortement influencés par les caractéristiques des institutions ${ }^{12}$.

En résumé, le paradigme institutionnaliste traditionnel postule que les caractéristiques des arrangements institutionnels déterminent les choix des individus tandis que le paradigme behavioriste classique postule que les choix dépendent des goûts et des valeurs des individus alors que le paradigme néo-behavioriste postule que les choix des individus dépendent à la fois des arrangements institutionnels et des goûts et valeurs des individus.

Par ailleurs, depuis la parution des ouvrages magistraux d'Anthony Downs et de Mancur Olson, les politologues ont été invités à ajouter à leurs analyses un troisième type de variables: les caractéristiques des biens collectifs ${ }^{13}$. D'après Mark SprouleJones,

«il faut considérer la nature du bien à produire avant de tirer des conclusions à propos des relations qui existent entre les arrangements institutionnels et les comportements des individus. De plus, il faut considérer les arrangements institutionnels pour comprendre la relation qui existe entre les individus et les biens à produire et, enfin, il est impossible de saisir cette relation sans considérer les préférences des individus ${ }^{14}$."

En conclusion, la science politique pourrait s'inscrire dans la trame d'un processus de développement cumulatif des connaissances en utilisant des méthodes de formalisation qui rendraient compte des relations entre les caractéristiques des arrangements institutionnels, les goûts et les valeurs des individus et les caractéristiques des biens à produire (graphique 1).

12. $\grave{A}$ titre d'exemples consulter William H. Riker, "Implications from Disequilibrium of Majority Rule for the Study of Institutions"; Kenneth A. Shepsle, "The Role of Institutional Structure in the Creation of Policy Equilibrium»; Russell Hardin, Collective Action (Baltimore: The Johns Hopkins University Press, 1982).

13. Antony Downs, An Economic Theory of Democracy, (New York: Harper and Row, 1957); Mancur Olson, La logique de l'action collective, (Paris: Presses universitaires de France, 1974), publié initialement en anglais en 1965.

14. Mark Sproule-Jones, «Public Choice Theory and Natural Resources: Methodological Explication and Critique», American Political Science Review, 76 (1982), 795. 
GRAPHIQUE 1

Illustration des éléments du programme de recherche de la science politique

caractéristiques des arrangements goûts et valeurs des individus

institutionnels

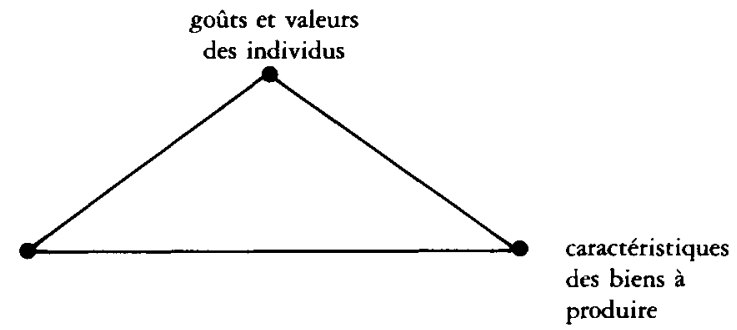

\section{Le contexte historique}

Les années dix-neuf cent quatre-vingt marquent l'émergence et la consolidarion d'un procès virulent à l'endroit des institutions politiques. D'après John Naisbitt, la création et le développement des institutions politiques du «welfare state» prennent leurs sources dans la crise économique des années dix-neuf cent trente qui provoqua une perte de confiance des individus dans leur capacité de résoudre par eux-mêmes leurs problèmes de santé, d'éducation et de survie économique ${ }^{15}$. C'est à partir de ce moment, soutient John Naisbitt, que

"We began to think that only with the strength of large institutions behind us we could effectively counter life's blows. More and more, we relied on government to provide for basic needs. Government's traditional function is to safeguard citizens. We also asked that it provide food, shelter, and jobs. But by the 1960s, the government's role had grown to testing toys and regulating the environment and much of the economy ${ }^{16}$."

Ces institutions ont failli à leurs tâches à plusieurs reprises. Par contre, un nombre croissant d'individus estime, depuis une

15. John Naisbitt, Megatrends, Ten New Directions Transforming Our Lives, (New York: Warner Books Inc., 1984), chapitre 6, 143-174.

16. John Naisbitt, Megatrends, 143-144. 
quinzaine d'années, que l'échec de nos institutions de production de biens collectifs est dramatique. La lutte contre le chômage et la pauvreté a été perdue; les services de santé sont d'une qualité douteuse alors que les institutions d'éducation produiraient des élèves dont la qualité de la formation serait de plus en plus discutable. Ainsi, par exemple, le dernier ouvrage de Mancur Olson soutient que l'action des groupes d'intérêt entraine la formation de coalitions redistributives qui accroissent la complexité de la règlementation, le rôle du gouvernement et affecte substantiellement la direction de l'évolution sociale ${ }^{17}$. D'après Lester Thurow, ce processus de redistribution crée souvent des situations de jeu à somme nulle:

«For most of our problems there are several solutions. But all these solutions have the characteristics that someone must suffer large economic losses. No one wants to volunteer for this role... Everyone wants someone else to suffer the necessary economic losses, and as a consequence none of the possible solutions can be adopted... Our political and economic structure simply ins't able to cope with an economy that has a substantial zero-sum element ${ }^{18}$."

Un jeu à somme nulle, faut-il le préciser, est un jeu où les pertes égalent exactement les bénéfices. Le problème fondamental tient au fait que les arrangements institutionnels actuels sont incapables de solutionner les problèmes d'allocation de coûts: par exemple, à quel endroit est-il rationnel de construire une route, une usine d'épuration d'eau, un corridor de transport d'électricité?

Chaque groupe d'intérêt tente de faire adopter les arrangements institutionnels qui le protègent contre les coûts externes engendrés par les décisions et les actions d'autres individus. Plus les arrangements institutionnels prévoient des mécanismes de compensation

17. Mancur Olson, The Rise and Decline of Nations, 36-74.

18. Lester C. Thurow, The Zero-Sum Society, (New York: Basic Books, Inc., 1980), 11. 
pour supporter les coûts externes, plus les groupes concernés sont incités à demander des mécanismes additionnels de protection, et plus le changement devient difficile à réaliser. C'est ainsi, par exemple, qu'une politique de stimulation de l'innovation technologique répond à des demandes de compensation à la fois du côté des entrepreneurs, qui veulent être compensés pour les risques financiers qu'ils prennent et, aussi, par les travailleurs, qui désirent être compensés pour les coûts possibles que l'innovation technologique entraîne sur leurs revenus. Les coûts de l'innovation technologique sont alors peut-être plus élevés que les bénéfices que la collectivité en retire. Aussi, le sénateur Maurice Lamontagne soutient-il que la quatrième révolution scientifique sera difficile à faire à cause du développement de contraintes institutionnelles défavorables. ${ }^{19}$ D'après John Cornwall et Wendy Maclean, il est grand temps que l'examen des politiques économiques tienne compte de l'impact des arrangements institutionnels;

«Historical developments leading to institutional changes in the nature of production, work, leisure and economic power relations cannot be ignored in studying any economic problem of significance. Advocates of the competitive model feel strongly that market forces will bring about definitive predictable and optimal forms of economic organization, whatever else has taken place historically, provided something called the price mechanism is allowed to free play. Hence efforts to study institutional changes are wasted efforts... The real world cannot be finessed, as it does not even behave "as if" atomistic competition prevails. The problems of inflation, stagnation and unemployment can only be understood and combatted when seen within the institutional context in which they arose. As institutions change, so do both economic behavior and the policies required to achieve agreed upon economic goals. One of the outstanding characteristics of the modern age has been the acceleration of institutional change. Nowhere is this more true than in the developments in the labour markets. The rise of trade unions, the greater sophistication and fabrication of goods, the growth of the welfare state and full employment

19. Maurice Lamontagne, Business Cycles in Canada, (Ottawa: Canadian Institute for Economic Policy, 1984), 158-168. 
policies have all acted to change markedly the workings of labour (and product) markets. New goals of economic activity and new contraints on realizing these goals develop. ${ }^{20}$."

En résumé, le nouvel intérêt porté à l'analyse des institutions origine à la fois de la perte de confiance dans les institutions publiques et de changements rapides et importants des arrangements institutionnels.

\section{Les arrangements institutionnels}

On l'a vu implicitement, le concept d'institution recouvre deux acceptions générales. Par institution, on peut entendre ou bien la structure d'une organisation et sa constitution écrite, ou bien les règles se rapportant à la prise de décision ${ }^{21}$. À considérer l'institution comme une unité indifférenciée on risque de parler de tout et donc de rien. L'institution ne se laisse pas saisir comme un ensemble unifié d'individus et de biens collectifs mais comme des ensembles d'individus poursuivant des buts plus ou moins conflictuels, accordant des valeurs différentes aux biens collectifs produits dans le cadre des institutions. L'analyse des choix collectifs exige donc qu'on désagrège le concept d'institution de façon à différencier les divers aspects des décisions et actions des individus ${ }^{22}$.

Le concept de règles permet de désagréger le concept d'institution. Le concept de règles doit cependant être précisé. Dans la théorie des jeux, les règles renvoient «aux instructions requises

20. John Cornwall et Wendy Maclean, Economic Recovery For Canada: A Policy Framework, (Ottawa: Canadian Institute for Economic Policy, 1984), 22.

21. Jacques Chevallier établit une distinction de ce type dans "L'analyse institutionnelle", dans Jacques Chevallier et autres, L'institution, (Paris: Presses universitaires de France, 1981), 3-61.

22. Richard Rose fait un plaidoyer similaire par rapport au concept de gouvernement dans «Disagregating the Concept of Government ", dans Charles Lewis Taylor (ed.), Why Governments Grow, Measuring Public Sector Size, (Beverly Hills, Ca.: Sage Publications, 1983), 157-176. 
pour participer au jeu» ${ }^{23}$; d'après Shubik, «the rules of the game include not only the move and information structure and the physical consequences of all decisions, but also the preference systems of all the players ${ }^{24}$.

Mais quel est donc au juste l'objet de ces règles? Bien qu'il ne s'agisse ici en rien d'une réponse définitive, on peut faire l'hypothèse que les règles d'un arrangement institutionnel renvoient au moins à onze types d'objets: 1) des règles qui identifient la nature des enjeux de décisions collectives, c'est-à-dire les objets sur lesquels les participants peuvent prendre des décisions; 2) Des règles qui précisent les types d'actions permises, notamment la façon dont les individus peuvent utiliser leurs ressources ${ }^{28}$; 3) Des règles qui déterminent la séquence des actions permises; 4) Des règles qui définissent les rôles des participants et le nombre de participants pour chaque rôle; 5) Des règles qui énoncent de quelle façon les participants sont sélectionnés pour remplir les rôles et la façon dont les participants abandonnent leurs rôles; 6) Des règles qui précisent la façon dont les participants peuvent se regrouper pour constituer des coalitions; 7) Des règles qui déterminent l'information disponible pour chacun des participants; 8) Des règles qui stipulent la façon d'agréger les décisions individuelles en choix collectifs. 9) Des règles qui déterminent la façon de partager le règlement des enjeux; 10) Des règles qui stipulent les actions à accomplir pour les participants qui veulent modifier une règle; 11) Des règles qui précisent le rôle des participants chargés d'assurer le respect des règles.

23. Il s'agit là de la définition de Steven J. Brams, dans Game Theory and Politics, (New York: The Free Press, 1975), 3.

24. Martin Shubik, Game Theory in the Social Sciences. Concepts and Solutions, (Cambridge, Mass.: The MIT Press, 1982), 8.

25. F. G. Bailey, Les règles du jeu politique, (Paris: Presses universitaires de France, 1971), 33-34. 
Contrairement à F.G. Bailey, cette conception des arrangements institutionnels tient compte des difficultés que pose l'agrégation des décisions individuelles en choix collectifs et des complications que pose l'imperfection de l'information à la disposition des individus ${ }^{25}$. La conception de F.G. Bailey postule implicitement l'inexistence de problèmes d'agrégation dans le passage des décisions individuelles aux choix collectifs. De plus, l'absence de règles concernant l'information à la disposition des individus équivaut à postuler implicitement, ou bien que l'information est parfaite, ou bien encore que les variations dans le degré d'information à la disposition des individus n'exercent aucun impact sur la prise de décision. La définition proposée récemment par Elinor Ostrom néglige, en dépit de son caractère très élaboré, de rendre compte des actions à entreprendre dans l'éventualité où une règle n'est pas suivie, ou encore, dans l'éventualité où l'on voudrait modifier une règle ${ }^{26}$. Finalement, le concept d'arrangements institutionnels proposé plus haut tient compte, à la suite d'Andrew Schotter, de la nécessité de règles d'autorité déterminant le rôle des participants chargés de veiller au respect des règles ${ }^{27}$.

\section{L'impact des arrangements institutionnels}

Les règles qui déterminent les arrangements institutionnels exercent un impact sur la production et la consommation de biens collectifs seulement si deux conditions sont satisfaites: il faut d'abord démontrer qu'une ou plusieurs règles d'un arrangement institutionnel amènent les participants à prendre des décisions

26. Elinor Ostrom, "An Agenda for the Study of Institution", adresse présidentielle à la Public Choice Society lors de sa réunion de mars 1984, 26.

27. Andrew Schotrer, The Economic Theory of Social Institutions, (Cambridge: Cambridge University Press, 1981), 11.

28. Cette règle pourrait être précisée en consultant les travaux sur la théorie des droits de propriété. 
qu'ils n'auraient pas prises sans l'existence de ces règles; il est ensuite nécessaire de démontrer que les décisions individuelles affectées par ces règles entraînent des résultats collectifs qui diffèrent de ceux qu'auraient amené d'autres règles.

De plus, toute tentative visant à démontrer l'impact des règles d'un arrangement institutionnel sur les décisions d'un individu et les résultats collectifs qui en découlent repose sur l'acceptation d'au moins quatre postulats: il faut d'abord supposer que les individus impliqués dans une situation de prise de décision connaissent parfaitement bien toutes les règles des arrangements institutionnels régissant la situation de prise de décision car, si ce n'était pas le cas, les décisions s'expliqueraient non par les caractéristiques des règles des arrangements institutionnels mais par le degré d'information dont disposent les participants. Il faut ensuite supposer que les individus impliqués dans la prise de décision comprennent de façon identique la façon d'utiliser les règles car, s'il en était autrement, les décisions varieraient d'un individu à l'autre non à cause des règles mais en raison de différences de compréhension qu'en auraient les participants. En troisième lieu, il faut supposer que les individus impliqués dans la prise de décision disposent de ressources égales parce que, si cela n'était pas le cas, les individus qui contrôleraient une quantité disproportionnellement élevée de ressources pourraient affecter la situation de prise de décision en faisant adopter des règles qui les avantageraient de façon décisive; d'autre part, même si les règles n'étaient pas modifiées, la situation de compétition parfaite définie par un partage égal des ressources se transformerait en une situation oligopolistique si seulement quelques individus contrôlaient une large proportion des ressources: la théorie économique prédit en effet que les décisions des producteurs en situation d'oligopole different de celles prises en situation de concurrence parfaite. Le quatrième postulat suppose que les individus accordent le même degré de légitimité aux règles des arrangements institutionnels. 
Si les individus accordaient un degré différent de légitimité aux règles, les décisions pourraient varier d'un individu à l'autre non pas à cause des caractéristiques des règles mais en raison de variations dans leur degré de légitimité.

Les règles des arrangements institutionnels comportent des mécanismes d'incitations et de contraintes qui exercent deux types d'impact sur les décisions individuelles ${ }^{29}$. Les règles des arrangements institutionnels contribuent à restreindre la gamme des actions possibles d'un individu en déterminant à l'avance les types d'actions permises et les types d'actions interdites. Ainsi, par exemple, les dirigeants d'un groupe d'intérêt, disons un syndicat, ne peuvent généralement pas hausser les cotisations des membres sans l'approbation d'une majorité. Les députés de l'Assemblée nationale du Québec n'ont pas le droit d'adopter des lois et règlements concernant les tarifs de la poste. D'autre part, les règles des arrangements institutionnels contribuent en même temps à accroître le degré de justesse des prédictions qu'un individu peut faire au sujet des décisions des autres individus impliqués dans une situation de décision: sachant que des règles restreignent les choix des autres individus, il devient plus facile de prédire correctement les actions qu'ils choisiront en réponse à nos propres actions. Ainsi, par exemple, un vendeur de meubles se sent plus libre de faire crédit à un client en sachant qu'il existe des règles garantissant le paiement ultérieurement et que le respect de ces règles est assuré par les règles qu'appliquent les policiers et les juges. L'absence de telles règles inciterait les vendeurs à conclure leurs transactions en exigeant le paiement comptant immédiat des biens vendus.

29. Cette question est traitée par Larry L. Kiser et Elinor Ostrom, "The Three Worlds of Action: A Metatheoretical Synthesis of Institutional Approaches» dans Elinor Ostrom (ed.), Strategies of Political Inquiry. (Beverly Hills, Calif. : Sage Publications, 1982), 191-192. 


\section{Méthodes d'évaluation de l'impact des arrangements institutionnels}

Les manuels de méthodologie n'abordent pas explicitement l'analyse des arrangements institutionnels ${ }^{30}$. Ils postulent implicitement que les décisions des individus dépendent uniquement de leurs goûts et de leurs valeurs alors qu'elles dépendent simultanément des goûts et des valeurs des individus, des règles des arrangements institutionnels et des caractéristiques des biens collectifs, En pratique, l'étude des décisions est toutefois grandement simplifiée si l'on vise à déterminer l'impact d'une variation d'un de ces trois facteurs alors que les deux autres ne se modifient pas. L'étude des décisions des individus peut prendre au moins huit grandes orientations (Tableau 1). Si les caractéristiques des biens collectifs et les règles des arrangements institutionnels sont fixes, les décisions des individus peuvent être expliquées ou prédites à partir des variations dans les goûts des individus (cellule FFV); d'autre part, si les caractéristiques des biens collectifs et les goûts des individus sont fixes, les décisions individuelles peuvent être expliquées ou prédites en se basant sur les variations des règles des arrangements institutionnels (cellule FVF); par ailleurs, si les règles des arrangements institutionnels et les goûts des individus sont fixes, les modifications de décision s'expliquent ou se prédisent à partir de changements dans les caractéristiques des biens collectifs (cellule VFF). Suivant cette perspective, les décisions des individus seront faciles à expliquer ou à prédire si les caractéristiques des biens collectifs, les règles des arrangements institutionnels et les goûts sont fixes (cellule FFF) parce qu'on n'observera alors aucun

30. On peut noter l'absence de références aux arrangements institutionnels en consultant des ouvrages tels que David Nachmias et Chavas Nachmias, Research Methods in the Social Sciences, Second Edition, (New York: St. Martin's Press, 1981); Benoît Gauthier, ed., Rechenche sociale. De la problématique à la collecte des données, (Sillery: Presses de l'Université du Québec, 1984). 
changement dans les décisions tandis que les modifications de décisions seront beaucoup plus difficiles à expliquer ou à prédire, si l'on se trouve dans un contexte où deux des trois facteurs se modifient simultanément (cellules FVV, VFV et VVF). La situation de décision la plus difficile à analyser est évidemment celle où un changement de décision peut dépendre de changements simultanés des caractéristiques des biens collectifs, des règles et des goûts (cellule VVV). L'étude de l'impact des règles des arrangements institutionnels sur les décisions des individus renvoie aux stratégies des cellules FVF, FVV, VVF et VVV. Il serait préférable de s'attaquer directement aux situations représentées dans les cellules FVV, VVF et VVV parce qu'elles correspondent vraisemblablement mieux à la réalité, mais il est plus prudent d'aborder l'étude de l'impact des arrangements institutionnels en supposant plus modestement que les règles changent alors que les caractéristiques des biens collectifs et les goûts des individus restent fixes (cellule FVF).

Par ailleurs, bien que le programme de recherche le plus simple consiste à étudier l'impact des règles en ne considérant

TABLEAU 1

Orientations possibles de l'analyse des décisions des individus

\begin{tabular}{|c|c|c|c|c|c|}
\hline \multirow{2}{*}{\multicolumn{2}{|c|}{$\begin{array}{l}\text { Caractéristiques } \\
\text { des biens } \\
\text { collectifs }\end{array}$}} & \multirow{2}{*}{\multicolumn{2}{|c|}{$\begin{array}{c}\text { Règles des } \\
\text { arrangements } \\
\text { institutionnels }\end{array}$}} & \multicolumn{2}{|c|}{ Goûts des individus } \\
\hline & & & & Fixes & Variables \\
\hline \multirow{2}{*}{ Fixes } & \multirow{2}{*}{ F } & Fixes & F & FFF & FFV \\
\hline & & Variables & $\mathrm{v}$ & FVF & FVV \\
\hline \multirow{2}{*}{ Variables } & \multirow{2}{*}{ v } & Fixes & $\mathrm{F}$ & VFF & VFV \\
\hline & & Variables & $\mathrm{v}$ & VVF & VVV \\
\hline
\end{tabular}


qu'une seule règle à la fois, il serait plus réaliste, suivant en cela l'avis d'Elinor Ostrom, d'étudier l'impact simultané de configurations de règles car les décisions des individus sont influencées non par une seule règle prise isolément mais par des configurations de règles ${ }^{31}$. Les travaux de Kenneth Shepsle et Barry Weingast tendent en effet à montrer que l'agrégation des décisions des individus produit plus souvent des décisions collectives cohérentes dans le cas où l'analyse considère l'effet simultané de plusieurs règles que dans ceux où l'on ne considère que l'impact d'une seule règle à la fois ${ }^{32}$.

Bien qu'il ne soit pas toujours possible de considérer les règles de façon indépendante, la stratégie méthodologique optimale consiste à étudier l'impact de configurations de règles sur les décisions des individus en faisant varier les règles une par une. La question à résoudre devient alors la suivante: quel est l'impact de la modification d'une règle sur les décisions d'un individu? Le meilleur devis de recherche consisterait à concevoir et à réaliser des expériences véritables. Un tel devis exigerait que les participants à une décision soient attribués de façon aléatoire à un groupe expérimental et à un groupe de contrôle. Le groupe expérimental serait celui dont les participants subiraient un changement de règles alors que les règles resteraient fixes pour les participants du groupe de contrôle. L'effet net d'une modification d'une règle d'un arrangement institutionnel s'obtient alors à l'aide de la formule suivante:

$$
\mathrm{I}_{\mathrm{n}}=\left(\mathrm{E}_{2}-\mathrm{E}_{1}\right)-\left(\mathrm{C}_{2}-\mathrm{C}_{1}\right)
$$

qui signifie que l'impact net $\left(\mathrm{I}_{\mathrm{n}}\right)$ d'une modification d'une règle égale la différence qui existe entre une décision prise après la

31. Cet argument est emprunté à Elinor Ostrom dans «An Agenda for the Study of Institutions", 19-22.

32. Kenneth A. Shepsle et Barry R. Weingast, "Structure-Induced Equilibrium and Legislature Choice», Public Cboice, 37 (1981), 503-520. 
modification d'une règle par rapport à une décision prise avant la modification de cette règle $\left(E_{2}-E_{1}\right)$ moins la différence qui existe entre une décision prise sans changement de règles à deux moments distincts $\left(C_{2}-C_{1}\right)$. Une représentation schématique d'un tel devis de recherche est présentée au tableau 2.

TABLEAU 2

Représentation d'un devis expérimental d'évaluation de l'impact d'une intervention sur les règles des arrangements institutionnels

\begin{tabular}{|l|c|c|}
\cline { 2 - 3 } \multicolumn{1}{c|}{} & $\begin{array}{c}\text { Avant la modification } \\
\text { d'une règle }\end{array}$ & $\begin{array}{c}\text { Après la modification } \\
\text { d'une règle }\end{array}$ \\
\hline $\begin{array}{l}\text { Groupe } \\
\text { expérimental }\end{array}$ & $\mathrm{E}_{1}$ & $\mathrm{E}_{2}$ \\
\hline $\begin{array}{l}\text { Groupe } \\
\text { de contrôle }\end{array}$ & $\mathrm{C}_{1}$ & $\mathrm{C}_{2}$ \\
\hline
\end{tabular}

Un devis de recherche de ce type fournirait de l'information sur les effets nets d'une modification d'une règle en permettant de contrôler l'impact de changements dans les caractéristiques des biens collectifs et goûts des individus. Un devis de recherche moins exigeant consisterait à évaluer les effets bruts de la modification d'une règle en utilisant la formule $I_{n}=\left(E_{2}-E_{1}\right)$, qui signifie que l'impact brut est égal à la différence entre une décision prise après une modification d'une règle par rapport à une décision prise avant une telle modification. Cette seconde formule se distingue de la première en ce qu'elle ne tient pas compte des changements dans les caractéristiques des biens collectifs et les goûts des individus (ceux-ci étant évalués par la différence $\left(C_{2}-C_{1}\right)$. Une telle stratégie reposant sur un devis de recherche de type expérimental paraît d'autant plus appropriée que les règles des arrangements institutionnels constituent des variables d'action qui peuvent être modifiées par les participants à une situation de décision. Les 
caractéristiques des biens collectifs et les goûts des individus sont beaucoup plus difficiles à modifier rapidement que les règles des arrangements institutionnels.

\section{Une illustration de l'évaluation de l'impact des arrangements institutionnels}

Soit une situation de décision où deux individus, $A$ et $B$, produisent chacun un bien, disons des pommes de terre pour $A$ et du bœuf pour B. Ces biens sont facilement mesurables parce qu'ils peuvent se mesurer en référence à une unité de mesure et à une quantité, divisibles, parce que les quantités produites constituent une somme finie qui peut être fractionnée entre A et $B$, et d'exclusion facile parce qu'un individu qui ne produit pas d'un bien ou ne l'achète pas peut être empêché de le consommer. Supposons aussi que $A$ et $B$, qui sont séparés par un fleuve large et profond, cherchent à maximiser les utilités qu'ils retirent de leur production de pommes de terre ou de bouf ${ }^{33}$. Dans un contexte où il y a rareté de ressources et absence de contacts entre $A$ et $B$ (à cause du fleuve...), ceux-ci ne peuvent augmenter leurs utilités qu'en travaillant davantage pour produire une plus grande quantité de pommes de terre et de bouf. Leur situation de décision se transforme radicalement s'ils entrent en contact par suite de la découverte d'un lieu où le fleuve est plus étroit et moins profond, donc moins dangereux à traverser. Si leurs actions ne sont limitées par aucune règle, on peut s'attendre à ce que les deux individus choisissent les actions qui maximisent leurs utilités. En conséquence, chacun est amené à voler l'autre jusqu'au point

33. Le mot "utilités» est pris dans le sens de satisfactions monétaires et non monétaires. 
où il retire plus d'utilités marginales de la prédation que par sa production personnelle de pommes de terre ou de bouf.

En même temps qu'ils estiment qu'il est rationnel d'investir dans la prédation, les individus $\mathrm{A}$ et $\mathrm{B}$ sont amenés à investir des ressources dans la protection de leur production (de même évidemment que dans la production qu'ils ont volée). On peut s'attendre à ce que leurs attaques et contre-attaques les amènent vers un état d'équilibre où chacun investit une quantité donnée de ressources pour la défense et la prédation et où chacun ne trouve profitable d'investir davantage de ressources dans la défense et la prédation. Il ne s'agit pas ici de se prononcer sur la désirabilité sociale d'un tel état d'équilibre mais uniquement de noter la dynamique de cette situation de décision.

Les ressources diverties de la production vers la prédation et la défense constituent en quelque sorte des ressources perdues. L'investissement de ces ressources dans la production de biens pourrait accroître le niveau d'utilités de $\mathrm{A}$ et $\mathrm{B}$. Les individus $\mathrm{A}$ et $\mathrm{B}$ sont donc dans une certaine mesure incités à adopter une règle qui restreint le comportement prédatoire, permettant ainsi de réduire la quantité de ressources qui doit être divertie de la production vers la défense. En fait, plus l'interaction entre $A$ et $B$ tendra à être conflictuelle, donc plus coûteuse pour chacun, plus les deux individus seront incités à adopter une règle visant à résoudre les problèmes de conflits récurrents.

Le tableau 3 indique les niveaux d'utilités que $\mathrm{A}$ et $\mathrm{B}$ retirent suivant qu'ils se soumettent ou non à une règle qui interdit la prédation. Ils peuvent décider de se soumettre tous les deux à la règle, ou encore, de la violer en commun. D'autre part, A peut décider de respecter la nouvelle règle alors que $\mathrm{B}$ la viole, ou bien, $B$ peut décider de respecter la règle tandis que $A$ la viole. Les individus $A$ et $B$ retirent davantage d'utilités en respectant tous les deux la règle (cellule $\mathrm{A}_{2}, \mathrm{~B}_{2}$ ) qu'en la violant (cellule $\left.A_{1}, B_{1}\right)$. Par contre, si $A$ respecte la règle mais que $B$ la viole 
(cellule $A_{2} B_{1}$ ), B retire encore davantage d'utilités, recevant alors 16 utilités au lieu de 10 , alors que $A$ sort perdant en recevant 8 utilités au lieu de 15 . Il en va de même si $B$ respecte la règle mais que $A$ ne le fait pas (cellule $A_{1}, B_{2}$ ), car $A$ retire dans ce cas 18 utilités contre 15 alors que $B$ en reçoit 5 plutôt que 10 comme c'était le cas dans la cellule $A_{2}, B_{2}$.

TABLEAU 3

Matrice d'utilités de A et B

\begin{tabular}{|c|c|c|c|}
\hline & & \multicolumn{2}{|c|}{ Individu $\mathrm{B}$} \\
\hline & & $\begin{array}{c}\text { viole } \\
\text { la règle } \\
\mathbf{B}_{1}\end{array}$ & $\begin{array}{c}\text { respecte } \\
\text { la règle } \\
B_{2}\end{array}$ \\
\hline \multirow{2}{*}{$\begin{array}{c}\text { Individu } \\
\text { A }\end{array}$} & $\begin{array}{c}\text { viole } \\
\text { la règle } \\
A_{1}\end{array}$ & $\begin{array}{c}10,7 \\
\left(\mathbf{A}_{1}, \mathrm{~B}_{1}\right)\end{array}$ & $\begin{array}{c}18,5 \\
\left(A_{1}, B_{2}\right)\end{array}$ \\
\hline & $\begin{array}{c}\text { respecte } \\
\text { la règle } \\
\mathrm{A}_{2}\end{array}$ & $\begin{array}{c}8,16 \\
\left(\mathrm{~A}_{2}, \mathrm{~B}_{1}\right)\end{array}$ & $\begin{array}{c}15,10 \\
\left(\mathbf{A}_{2}, B_{2}\right)\end{array}$ \\
\hline
\end{tabular}

Au total chacun est donc incité à violer la règle parce que cela lui permet d'accroître son gain. Si A et B raisonnent de la sorte, le règlement qui surviendra correspondra aux utilités de la cellule $A_{1}, B_{1}$, soit 10 utilités pour $A$ et 7 pour $B$.

La cellule $A_{1}, B_{1}$ finit par constituer le règlement du conflit parce qu'aucun des individus accepte de se soumettre à la nouvelle règle dans une situation où l'autre peut décider de ne pas la respecter. La possibilité de faire défection de l'un incite l'autre à faire de même parce que $A$ retire plus d'utilités du règlement de la cellule $A_{1}, B_{1}$ que de la cellule $A_{2}, B_{1}$ et que $B$ retire lui-même davantage du règlement $A_{1}, B_{1}$ que du règlement $A_{1}, B_{2}$. Bref, $A$ et $B$ sont incités à revenir à la décision qui prévalait antérieurement 
à l'adoption de la règle pour des raisons purement défensives. Beaucoup de conflits, qu'ils soient internationaux, nationaux, locaux ou même conjugaux, évoluent vers la cellule $A_{1}, B_{1}$ parce qu'une partie craint que l'autre attaque en premier (ou viole en premier une règle) pour obtenir un gain plus élevé. Cette situation correspond à un exemple du dilemme du prisonnier.

La violation de la règle discutée plus haut peut être rendue beaucoup plus difficile par suite de l'adoption de règles qui identifient des individus chargés de faire respecter les règles (règle de type 11) et les actions que ces individus doivent accomplir lorsqu'une règle est violée (règle de type 10$)^{34}$. En termes concrets, cela pourrait signifier la création d'un appareil juridique et d'un corps policier. L'implantation d'un mécanisme surveillant et contrôlant l'obéissance à une règle peut entraîner des coûts élevés mais, ceuxci peuvent être plus petits que les coûts engendrés par le retour à la situation de décision antérieure à l'adoption de la règle antiprédatoire. Les coûts résultant de l'application du mécanisme contrôlant l'obéissance à une règle doivent faire l'objet d'un examen particulier. Supposons toutefois pour l'instant que la création d'un tel mécanisme produit davantage de bénéfices qu'elle n'engendre de coûts.

Si l'arrangement institutionnel ne prévoit pas de règles coercitives (règles de type 10) pénalisant les individus qui accomplissent des actions interdites (règles de type 2), A et $B$ investiront dans la production, la prédation et la défense (cellule $A_{1}, B_{1}$ ) obtenant ainsi un certain niveau d'utilités représenté par les points $U_{A 1}$ et $\mathrm{U}_{\mathrm{B} 1}$, ou par le point $\mathrm{X}$ du graphique 2. L'adoption d'une règle de type 10 force $A$ et $B$ à se déplacer vers la cellule $A_{2}, B_{2}$, augmentant ainsi les utilités de $A$ de $U_{A 1}$ à $U_{B 2}$ et celles de $B$ de $U_{B 1}$ à $U_{B 2}$ ou encore forçant les individus à se déplacer du point $\mathrm{X}$ vers le point $\mathrm{Y}$.

34. Il s'agit des types de règles introduits dans la section définissant le concept d'institution. 
GRAPHIQUE 2

Représentation des utilités

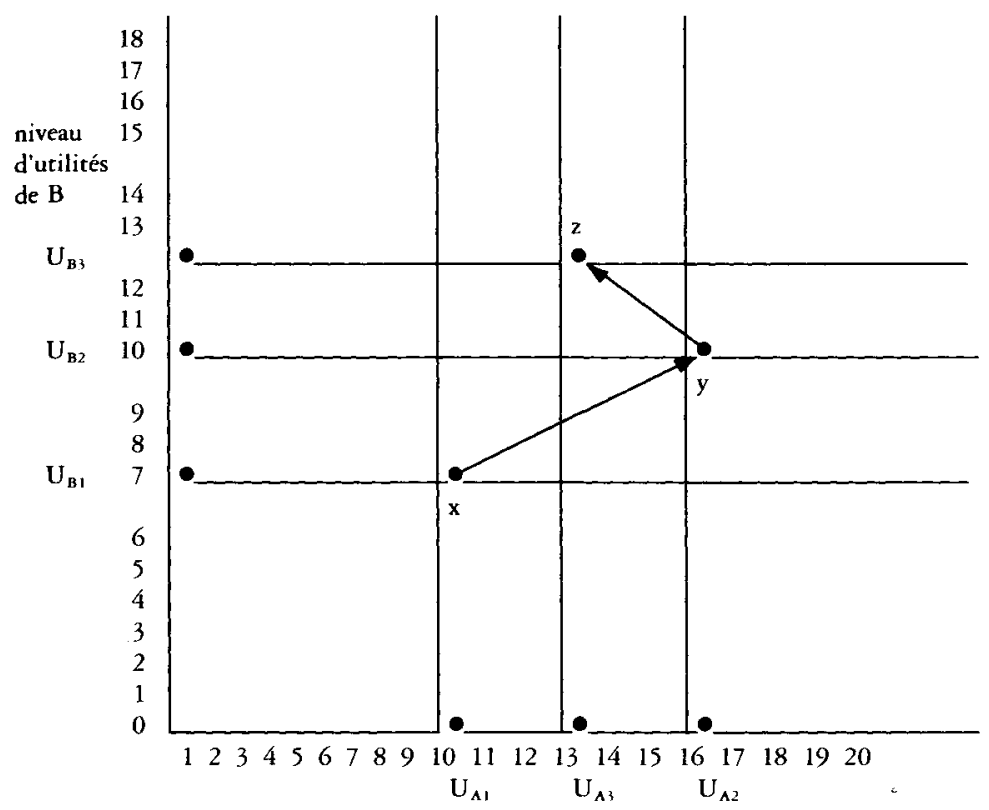

niveau d'utilités de $\mathrm{A}$

Cet exemple démontre que si les caractéristiques des biens produits et les goûts des individus ne changent pas, les décisions des individus sont modifiées non par suite de règles qui définissent les types d'actions permises (règles de type 2) mais par l'introduction de règles d'autorité (règles de type 10 et 11 ). Bref, la modification de la décision des individus $A$ et $\mathbf{B}$ résulte non pas d'une modification de leurs goûts ou des caractéristiques des biens produits mais de 
la modification de règles définissant les arrangements institutionnels ${ }^{35}$.

Par ailleurs, le règlement $\left(A_{2}, B_{2}\right)$ engendre une situation d'inégalité qui poussera l'individu désavantagé, en l'occurrence $B$ (qui reçoit 10 utilités alors que $A$ en retire 15 ) à tenter d'améliorer sa position tandis que $A$ tentera de préserver le statu quo. On peut prédire que $B$ tentera de faire modifier les règles de type 9 , qui déterminent la façon de partager le règlement, de façon à déplacer le règlement du point $Y$ au point $Z$ (Graphique 2$)^{36}$. L'exemple le plus familier est certainement celui des francophones du Québec qui demandent que les règles du fédéralisme canadien soient modifiées de façon à ne plus les désavantager alors que les Anglo-canadiens refusent de modifier les règles en prétendant qu'elles produisent des résultats équitables.

La durabilité du règlement $\left(A_{2}, B_{2}\right)$ dépend des coûts associés à l'application des règles de typos 10 et 11 , notamment des coûts résultant de l'implantation de mécanismes de contrôle tels que la création d'un corps policier ou la mise en place d'un appareil juridique, mais aussi des coûts découlant des sanctions imposées à la suite de violations d'une règle. Aussi peut-on suggérer les hypothèses suivantes:

- plus les coûts d'implantation et de fonctionnement du mécanisme de contrôle des règles seront élevés, plus la durabilité d'un règlement tel que $\left(A_{2}, B_{2}\right)$ sera courte;

- plus les coûts des sanctions imposées lors de la violation d'une règle seront élevés, plus la durabilité d'un règlement tel que $\left(A_{2}, B_{2}\right)$ sera longue;

35. Robert Axelrod, The Evolution of Cooperation, (New York: Basic Books Inc., 1984) démontre qu'il est très difficile de faire émerger la coopération dans un monde d'égoöstes où les individus ne sont contraints par aucune règle d'autorité.

36. Ce type de problème est traité de façon fort intéressante dans Edna UIImanMorgalit, The Emergence of Norms, (Oxford: Oxford University Press, 1977), 134-197 et Andrew Schotter, The Economic Theory of Social Institutions, 20-51. 
- plus le nombre de personnes impliquées dans une situation de décision sera grand, plus les coûts d'implantation et de fonctionnement du mécanisme de contrôle des règles seront élevés, plus la probabilité de détecter les violations d'une règle diminuera et, conséquemment, plus la durabilité d'un règlement tel que $\left(A_{2}, B_{2}\right)$ s'écourtera. Il s'agit ici d'une application de la loi d'Olson qui énonce qu'il est plus facile de faire cavalier libre (free rider) dans un grand groupe que dans un petit groupe ${ }^{37}$.

De plus, un règlement tel que $\left(A_{1}, B_{2}\right)$ aura une plus grande durabilité si les bénéfices des biens produits sont divisibles et d'exclusion facile, comme c'est le cas pour la production de bœuf et de pommes de terre, alors que, à l'inverse, un règlement tel que $\left(A_{2}, B_{2}\right)$ aura une durabilité plus courte si les bénéfices des biens produits sont indivisibles et d'exclusion difficile, comme ce serait le cas pour la dépollution de l'air et l'assainissement des eaux. Un règlement tel que $\left(A_{2}, B_{2}\right)$ aura moins de durabilité si les bénéfices du bien produit sont susceptibles de congestion, c'est-à-dire que les bénéfices des consommateurs commencent à décroître au-delà d'un seuil donné, alors qu'un règlement tel que $\left(A_{2} B_{2}\right)$ aura plus de durabilité si ses bénéfices sont non susceptibles de congestion.

De plus, un règlement tel que $\left(A_{2}, B_{2}\right)$ aura plus de durabilité dans un contexte d'incertitude, c'est-à-dire d'information imparfaite, si les coûts de production d'un bien sont faibles que dans une situation d'information imparfaite où les coûts de production sont élevés.

Finalement, la durabilité d'un règlement tel que $\left(A_{2}, B_{2}\right)$ dépend aussi de l'amplitude de l'écart qui sépare les bénéfices que reçoivent $A$ et $B$. Plus l'amplitude de cet écart sera élevée plus la durabilité d'un règlement sera brève. En second lieu, plus le

37. Mancur Olson, La logique de l'action collective, chapitre 2. 
degré de légitimité de l'écart sera faible, moins le règlement aura de durabilité. Par contre, dans une situation de jeu à somme nulle, plus les coûts de réduction d'un écart seront élevés, plus le rétrécissement de son amplitude est improbable (moins il est probable de passer du point $Y$ au point $Z$ sur le graphique 2), et plus un règlement tel que $\left(A_{2}, B_{2}\right)$, soit le point $Y$ du graphique 2 , aura de durabilité.

Kenneth Shepsle et Barry Weingast soutiennent que la durabilité d'un règlement dépend aussi d'événements non anticipés pouvant arriver d'une période à une autre, notamment d'une élection à une autre, et de la complexité des arrangements institutionnels, particulièrement de l'existence de plusieurs niveaux de juridictions ${ }^{38}$. La présence de ces deux types de facteurs accroît les coûts d'application des règles de type 10 et 11 et, conséquemment, écourte la durabilité d'un règlement

\section{Conclusion}

L'étude des règles des arrangements institutionnels est en train de devenir un sujet de plus en plus importants dans les théories politiques récentes concernant les groupes d'intérêt, le vote et la bureaucratie ${ }^{39}$. La science politique est en train de redevenir la science des institutions parce que l'analyse des décisions des individus impliqués dans la production de biens collectifs

38. Kenneth A. Shepsle et Barry R. Weingast, "When Do Rules of Procedure Matter?", The Journal of Politics, 46, (1984), 215.

39. À titre d'exemples sur les groupes d'intérêts, consulter Terry M. Moe, The Organization of Interests, Incentives and the Internal Dynamics of Interest Groups, (Chicago: The University of Chicago Press, 1980; sur le vote consulter: William H. Riker, Liberalism Against Populism: A Confrontation Between the Theory of Democracy and the Theory of Social Choice, (San Francisco: W.H. Freeman and Company, 1982); sur la bureaucratie consulter la synthèse de Xavier Greffe, Analyse économique de la bureaucratie, (Paris, Economica, 1981). Pour une application à une politique gouvernementale concernant le Québec, consulter Réjean Landry, «L'hydro-électricité du Québec: produire pour consommer ou produire pour exporrer?", Études internationales, 15, (1984), 95-120. 
démontre que les décisions des individus sont affectées non seulement par les goûts et les valeurs individuelles mais qu'elles sont également très sensibles aux règles définissant les arrangements institutionnels.

Le passage du paradigme behavioriste à un paradigme néobehavioriste qui réincorpore les arrangements institutionnels s'opère dans un contexte épistémologique nouveau qui situe la science politique dans un processus de développement cumulatif des connaissances plutôt que dans la trame des révolutions scientifiques. De plus, l'intérêt nouveau qu'on porte à l'étude des institutions s'insère dans un contexte historique particulier marqué d'une perte de confiance dans les institutions publiques et de changements rapides et importants dans les règles des arrangements institutionnels.

La nouvelle analyse institutionnelle définit le concept d'institutions de façon à saisir la diversité des aspects des décisions et la diversité des goûts des individus. Un arrangement institutionnel renvoie à un ensemble de règles qui caractérisent les situations de décision en référence à onze dimensions particulières. L'analyse de l'impact des arrangements institutionnels sur les décisions des individus et sur les choix collectifs qui en résultent peut se réaliser en adoptant un devis de recherche de type expérimental qui compare les différences dans les décisions des individus suivant qu'elles sont prises avant ou après l'introduction de règles spécifiques. Cette stratégie de recherche mérite d'autant plus d'attention que les règles des arrangements institutionnels constituent des variables d'action qui peuvent être modifiées par l'intervention des individus. L'illustration simple présentée dans la dernière partie de l'article démontre la grande sensibilité des décisions aux variations de certaines règles des arrangements institutionnels. L'analyse des caractéristiques de cette illustration débouche sur la formulation d'hypothèses concernant la durabilité du règlement d'un enjeu par suite de variations dans les caractéristiques des coûts et des bénéfices des biens produits et de modifications de diverses com- 
posantes des règles des arrangements institutionnels, notamment du degré d'information à la disposition des individus.

La nouvelle analyse institutionnelle risque de s'imposer très lentement parmi les politologues en raison du recours à la modélisation mathématique et de la position épistémologique de ce type d'analyse qui repose sur le postulat que l'unité d'analyse réside dans la décision de l'individu plutôt que du côté de collectivités comme la classe sociale, l'État ou la nation. Par contre, le manque d'intérêt des politologues à l'endroit des arrangements institutionnels est en train d'être comblé par les économistes qui commencent de plus en plus à considérer les décisions des individus en tenant compte non seulement des goûts individuels et des caractéristiques des biens collectifs mais aussi de l'impact décisif qu'exercent souvent les règles des arrangements institutionnels. 